\title{
Dinâmica de sistemas para modelagem de reatores em bateladas sequenciais
}

\author{
System Dynamics for sequencing batch reactors modeling \\ Fausto Alfredo Canales', Carlos André Bulhões Mendes² \\ 1,2 Departamento de Planejamento e Gestão de Recursos Hídricos, Instituto de Pesquisas Hidráulicas, \\ Universidade Federal do Rio Grande do Sul, Porto Alegre, Brasil.
}

\section{Resumo}

O tratamento de esgoto por reatores em bateladas sequenciais é uma variação do processo de lodos ativados, porque combinam todas as etapas e processos de tratamento em um único tanque. Neste trabalho, apresenta-se um modelo baseado em dinâmica de sistemas, criado no Vensim, que tem por objetivo simular o enchimento e esvaziamento dos tanques de um sistema de tratamento, por reatores em bateladas sequenciais, considerando grandes variações no regime de vazões afluentes ao longo do dia. O sistema representado está formado por dois tanques, um by-pass e, opcionalmente, uma bacia de amortecimento. Alguns exemplos são incluídos para ilustrar a capacidade do modelo de simular diferentes configurações do sistema, o que é possível verificar com base nos resultados dos exemplos mostrados. O modelo pode ser adaptado a fim de incluir características próprias de sistemas de tratamento similares, permitindo avaliar particularidades em função das características do esgoto afluente e propriedades físicas ou químicas da estação de tratamento.

Palavras-chave: Dinâmica de sistemas, Reatores em bateladas sequenciais, Vensim

\begin{abstract}
Wastewater treatment by sequencing batch reactors is a variation of the activated-sludge process, because they combine all of the treatment steps and processes into a single tank. This paper presents a model created in Vensim and based on system dynamics with the aim of simulating the filling and depletion of the tanks in a wastewater treatment system by sequencing batch reactors, considering a high variability of the inflow along the day. The system shown is comprised of two tanks, a bypass, and an optional influent equalization basin. Some examples are included to illustrate the capacity of the model to simulate different system configurations, something that is supported by the results of these examples. The model can be adapted to include characteristics of similar wastewater treatment systems, allowing the evaluation of different conditions depending on the characteristics of wastewater inflows and physical or chemical features of the treatment plant.
\end{abstract}

Keywords: System dynamics, Sequencing batch reactors, Vensim 


\section{INTRODUÇÃO}

O tratamento de esgoto pelo processo de lodos ativados, com operação em bateladas, realiza-se no interior de apenas um tanque ou reator. A diferença entre os reatores em bateladas sequenciais (RBS) e as estações convencionais de tratamento por lodos ativados é que os primeiros combinam todos os passos do tratamento em um único tanque, enquanto que as segundas precisam de múltiplas estruturas. Por esta razão, a United States Environmental Protection Agency (EPA, 1999) descreve os RBS como uma estação de tratamento por lodos ativados que atua no tempo em lugar do espaço.

Fazendo uso do apresentado por Santos et al. (2006), na Figura 1 se representa, esquematicamente, um reator em bateladas com suas diferentes zonas. Estes autores mencionam que o reator sequencial funciona, inicialmente, como tanque de aeração e, em seguida, como decantador final. Al-Rekabi et al. (2007) descrevem o processo básico de operação dos RBS, indicando que o tratamento do esgoto se realiza depois do período de tempo necessário para o enchimento dos tanques. Uma vez finalizado este procedimento, se permite a sedimentação do volume tratado e, posteriormente, o volume clarificado é retirado do tanque e liberado no corpo receptor, para permitir o início de um novo ciclo (batelada). A partir da informação encontrada em Novotny et al. (1989), na Figura 2 se apresenta a sequência operacional típica de um RBS. O volume de oxigênio fornecido e os tempos de cada fase devem ser suficientes para permitir uma correta biodegradação do substrato, a fim de atender os requerimentos ambientais aplicáveis.

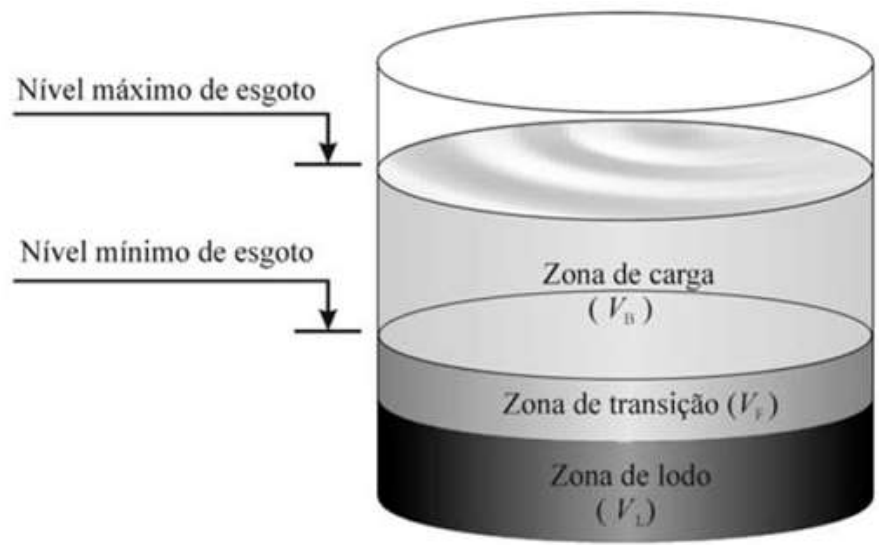

Figura 1 - Representação esquemática do reator em bateladas

Fonte: Fonte: Santos et al., 2006

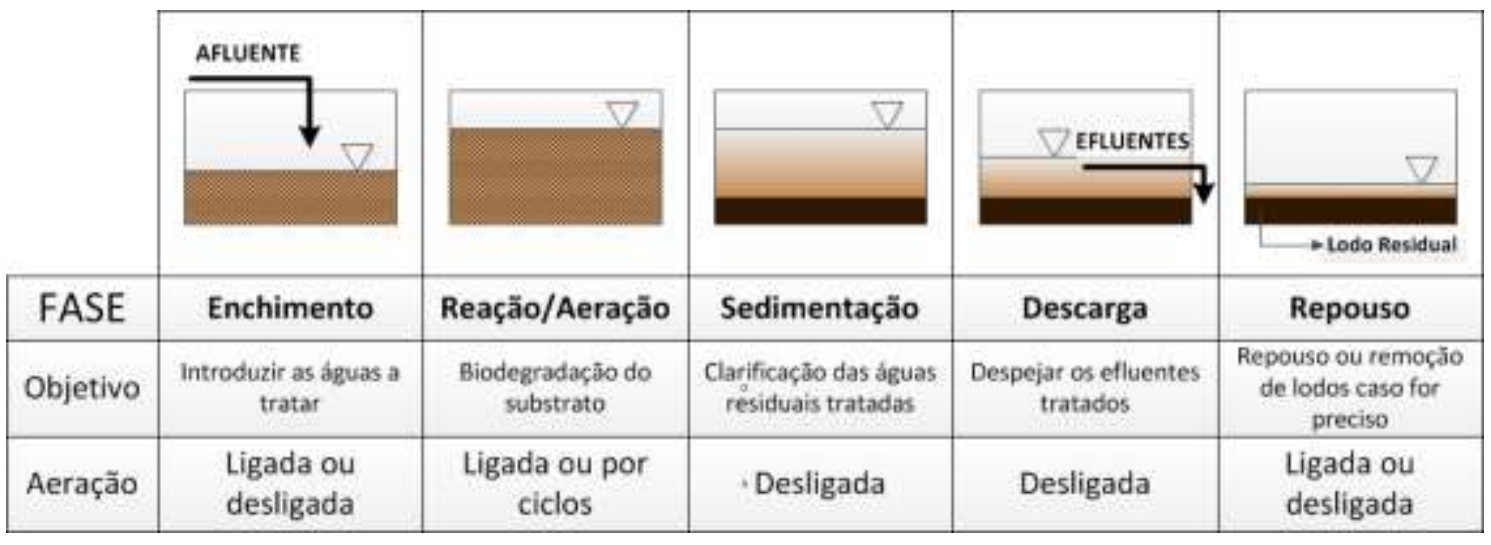

Figura 2 - Sequencia operacional típica de um Reator em Bateladas Sequenciais

Fonte: Adaptado de Novotny et al., 1989 
Em função do tipo de usuários atendidos, o regime de vazão de esgoto afluente a uma estação de tratamento com RBS pode apresentar, entre suas características temporais, a não uniformidade, com períodos de vazão nulos e grandes ou múltiplos picos que afetam os tempos de cada ciclo. Nesse contexto, a dinâmica de sistemas pode resultar uma ferramenta útil na representação das bateladas sequenciais, devido a que permite modelar e entender o comportamento temporal de sistemas complexos.

Segundo o explicado no trabalho de Izquierdo et al. (2008), a dinâmica de sistemas estuda variáveis observáveis de sistemas complexos, identificando as relações de causalidade, normalmente circulares, que existem entre elas. Muito frequentemente, os modelos da dinâmica de sistemas podem expressar-se, sem muita dificuldade, como um conjunto de equações algébricas (principalmente equações diferenciais), e suas variáveis são propriedades geralmente macroscópicas do sistema representado.

Entre os programas de software mais amplamente utilizados para criar modelos baseados em dinâmica de sistemas, pode-se mencionar: Stella, Simulink, Vensim e Powersim. Ford (2009) explica que os modelos podem ser vistos como uma coleção de equações diferenciais de primeira ordem, com uma equação diferente para cada variável de acumulação. As equações necessárias para criar modelos realistas são quase sempre não lineares, portanto faz sentido resolver as equações através da diferenciação e a integração numérica. Os programas, citados anteriormente, têm uma interface gráfica, o que permite a criação e o desenvolvimento de modelos com clareza visual. O Vensim foi empregado no presente trabalho para desenvolver o modelo proposto, por causa da facilidade na aprendizagem, e pela capacidade de simular o comportamento dinâmico com maior facilidade e nitidez visual, características também mencionadas por Ford (2009).

No âmbito dos recursos hídricos e de tratamento de águas, o Vensim tem sido aplicado em vários trabalhos, como os de Stave (2003), Liu et al.(2008), Gonçalves (2009) e Wei et al. (2012), entre outros. No relacionado, especificamente, ao uso do Vensim para analisar processos em RBS, pode-se salientar o trabalho de Souza (2011), que incluiu a avaliação de condições não estacionárias nas características químicas e da vazão do esgoto tratado, a fim de examinar os efeitos nos efluentes de um sistema de dois tanques sem bacia de amortecimento. No modelo em Vensim proposto por Souza (2011), o tempo que demoram os tanques em atingir seu volume máximo de carga está em dependência de uma vazão de projeto constante, situação que não consideraria adequadamente períodos de vazão nula ou picos ao longo do dia. O critério de adotar uma vazão constante para RBS é comum na literatura, como é possível observar nos exemplos do capítulo 8 de Metcalf \& Eddy (2003), em Santos et al. (2006) ou EPA (1999).

Com base no anterior, o objetivo do presente trabalho é apresentar um modelo, criado no Vensim, para simular o enchimento e o esvaziamento de um sistema de tratamento, por reatores em bateladas sequenciais, considerando grandes variações no regime de vazões afluentes ao longo do dia. O sistema representado está formado por dois tanques, um by-pass e, opcionalmente, uma bacia de amortecimento. A fim de validar e ilustrar a aplicabilidade do modelo, três exemplos são apresentados, considerando diferentes configurações do sistema. O modelo foi desenvolvido com o intuito de admitir modificações posteriores que permitam representar, mais plenamente, todos os processos unitários que possam ocorrer em uma determinada estação de tratamento com RBS.

\section{MÉTODO: DESCRIÇÃO DO MODELO}

Nesta seção se apresentam as equações, inequações e considerações, utilizadas do algoritmo representado no Vensim, para modelar o enchimento e o esvaziamento de reatores, em bateladas sequenciais, para tratamento de esgoto. Mesmo que o modelo aqui apresentado foi realizado na versão profissional do Vensim, com licença para a UFRGS, o modelo pode ser reproduzido perfeitamente na versão PLE, disponível gratuitamente no site http://vensim.com/, para propósitos acadêmicos e de avaliação.

\section{I Considerações do sistema modelado}

O sistema representado foi criado com base em uma estação de Recuperação da Qualidade Ambiental existente, descrita em Lopes (2011), a qual compreende um reator sequencial em batelada que consiste em obras de tratamento preliminar (gradeamento e remoção de areia), um tanque de aeração/decantação com seus correspondentes leitos de secagem de lodos e um by-pass para desvio 
de vazões excedentes. A estação foi projetada para atender as cargas diurnas, já que durante a noite e nos sábados, domingos e feriados não existem efluentes consideráveis, apenas alguma contribuição devida a águas de infiltração.

As obras existentes e a área na que estão inseridas foram projetadas de forma que permitiriam a construção, no futuro, de pelo menos mais um tanque, leitos de secagem adicionais e uma bacia de amortecimento, em função do crescimento da população atendida.

Com relação à bacia de amortecimento, a New England Interstate Water Pollution Control Commission (NEIWPCC, 2005) indica que o amortecimento é essencial onde existem ou se esperam variações significativas na magnitude das vazões afluentes ou nas cargas orgânicas, assim como quando é desejado realizar processos de nitrificação e desnitrificação. Igualmente, nesse texto se mencionam algumas vantagens do amortecimento em RBS:

- Permite um tamanho menor dos tanques, devido a que permitem o armazenamento até que o ciclo é completado.

- Permite a saída de um dos tanques para trabalhos de manutenção ou por variações sazonais, minimizando os custos associados.

- Permite a remoção de espumas e gorduras em um único local antes do seu ingresso ao tanque de aeração/decantação.

As principais considerações incluídas na modelagem do sistema representado no Vensim são as seguintes:

1. Existência de 1 ou 2 reatores (tanques). Quando seja avaliado o uso de um único reator, o valor do volume de enchimento (Fill.Vol1) do tanque 1 deverá ser fixado em $0,001 \mathrm{~m}^{3}$. O volume de enchimento é o volume correspondente à zona de carga do reator.

2. Capacidade de até duas bateladas por dia para cada reator.

3. Período máximo de simulação de sete dias (168 horas).

4. Existência opcional de uma bacia de amortecimento, a qual acumulará enquanto os tanques estiverem cheios ou esvaziando o volume tratado. $\mathrm{O}$ volume da bacia de amortecimento ( $\mathrm{CapBa}$ sin) deverá ser inferior ao volume de enchimento do tanque 2 (Fill.Vol2). Caso seja considerada uma bacia de amortecimento, o volume, nela armazenado até antes de iniciar o enchimento para a segunda batelada no tanque 2 , será adicionado durante este processo. O volume armazenado nela depois deste período será o volume inicial da primeira batelada no dia seguinte. 5. Uma vez que a bacia de amortecimento estiver cheia, a vazão excedente será despejada através do by-pass.

6. Se o tanque 2 não atinge seu volume máximo ao final do dia, considera-se que o volume com que se finaliza o dia é tratado e liberado antes do início do dia seguinte.

7. O modelo representado permite avaliar diferentes cenários a partir de modificações nos valores dos volumes de enchimento (Fill.Vol1, Fill.Vol2), volume da bacia de amortecimento (CapBasin), tempos de aeração e decantação $(t a, t d)$ e capacidade das bombas para retirada do efluente tratado $(D P R)$.

\subsection{DESCRIÇÃO DO MODELO}

O modelo em Vensim, aqui descrito, simula o enchimento e o esvaziamento dos tanques de um reator sequencial em batelada, segundo os parâmetros e as considerações mencionadas anteriormente. No Vensim foi criada uma vista para cada um dos sete dias que pode durar o período simulado, uma delas é mostrada na Figura 3. Nesta Figura, o sufixo -n (neste caso -3) indica o dia representado na vista.

Os elementos representados na Figura 3 e as fórmulas ou considerações utilizadas para determinar os respectivos valores são descritos a seguir:

Vazão afluente:

- QinTime $\left[m^{3} / h\right]$ : Esta é a vazão afluente ao sistema para um período de 24 horas, em função do dia correspondente. Para o presente trabalho, foi utilizado o mesmo hidrograma de vazões afluentes para todos os dias, o qual se mostra na Figura 4. Neste, foram considerados dois picos, por volta do horário das refeições, e vazões nulas, entre as 23 hs e 5 hs da manhã. Para 
ingressar os dados de vazão no modelo foi aproveitada a capacidade do Vensim de importar dados desde uma planilha no Excel.

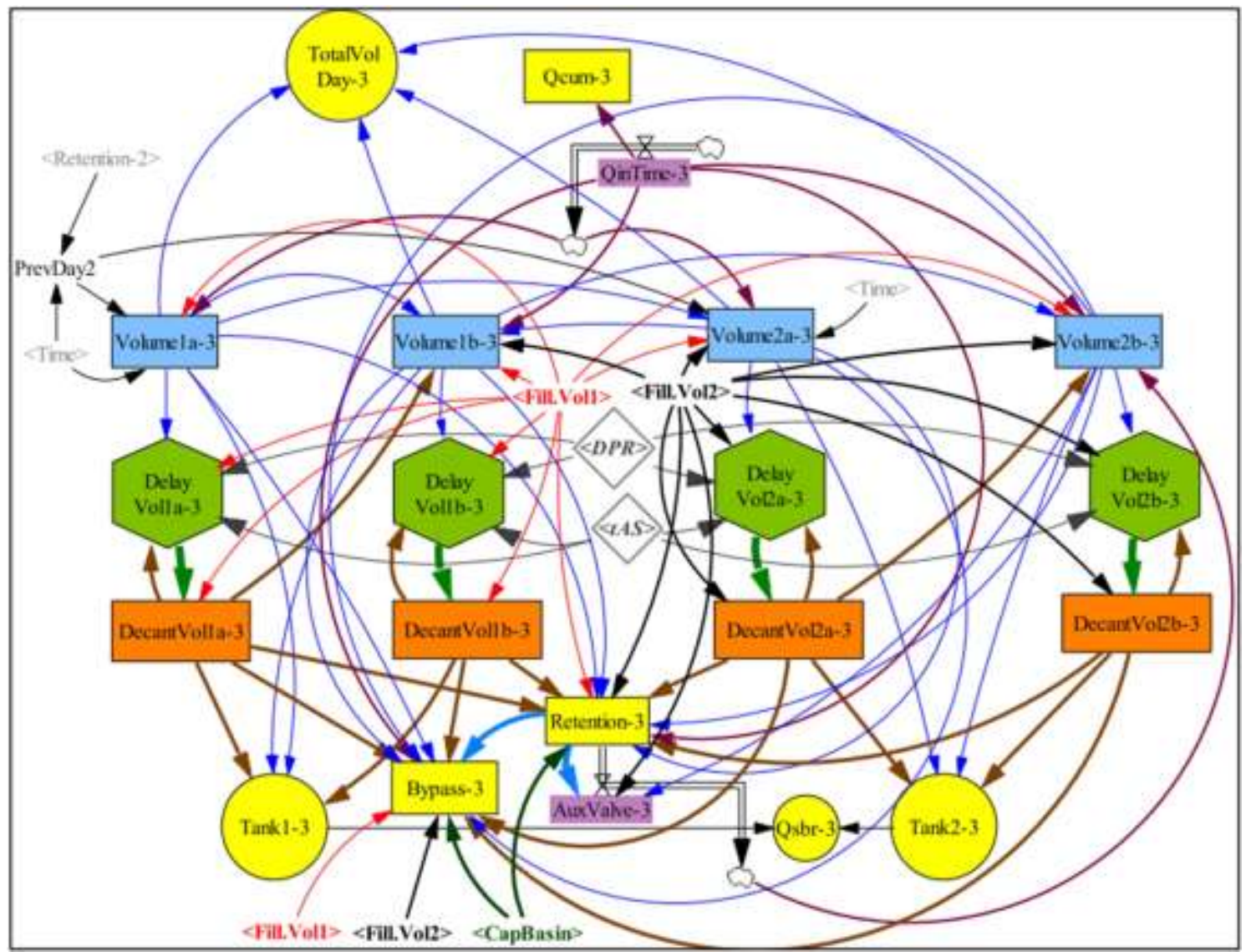

Figura 3 - Vista no Vensim das relações para simulação de um dia

- Qcum [m³]: Vazão afluente acumulada durante o dia correspondente. Este valor é utilizado para verificação e referência. Neste trabalho, seu valor para cada dia é de $1940 \mathrm{~m}^{3}$, obtido a partir da integração do QinTime.

Variáveis que definem o sistema: A seguintes são variáveis que podem ser modificadas com o propósito de avaliar diferentes cenários e, assim, poder realizar análises de sensibilidade:

- FillVoll e FillVol2 [m³]: Volumes da zona de carga dos reatores 1 e 2 . No caso de considerar a existência de um único tanque, o valor do FillVol1 deverá ser fixado em $0,001 \mathrm{~m}^{3}$.

- CapBasin [m³]: Volume da bacia de amortecimento.

- $t A S[h]$ : Tempo necessário para aeração e sedimentação do esgoto tratado. Isto constitui o tempo de retardo entre o momento que um reator completa seu volume de carga (FillVol1 ou FillVol2) e o início da retirada do efluente tratado.

- DPR $\left[\mathrm{m}^{3} / \mathrm{h}\right]$ : Capacidade das bombas para retirada do efluente tratado.

Bateladas: De forma geral, a modelagem do enchimento e esvaziamento dos reatores atende a seguinte a sequência lógica:

1. O volume na zona de carga com que inicia o reator 1 será o volume armazenado na bacia de amortecimento no final do dia anterior (PrevDay).

2. O reator 1 admitirá nele o armazenamento da vazão afluente até completar seu enchimento, dando início ao período de aeração e sedimentação da primeira batelada no reator 1 . O efluente tratado será retirado uma vez que tenha completado este período, concluindo assim a primeira batelada. 
3. Para a primeira batelada do reator 2 , este só começa a encher uma vez que se tenha completado o enchimento do reator 1. Igualmente, uma vez que o tanque 2 estiver cheio, ocorrem os processos de aeração, sedimentação e posterior esvaziamento do efluente tratado.

4. Depois do enchimento do reator 2, a segunda batelada no reator 1 só poderá iniciar até que finalize o esvaziamento do efluente tratado nesse tanque durante a primeira batelada. Caso contrário, e enquanto finaliza o esvaziamento do reator 1 , o armazenamento é realizado na bacia de amortecimento (retention), ou em caso desta não existir ou se tiver completado seu enchimento, o esgoto não tratado é despejado através do by-pass.

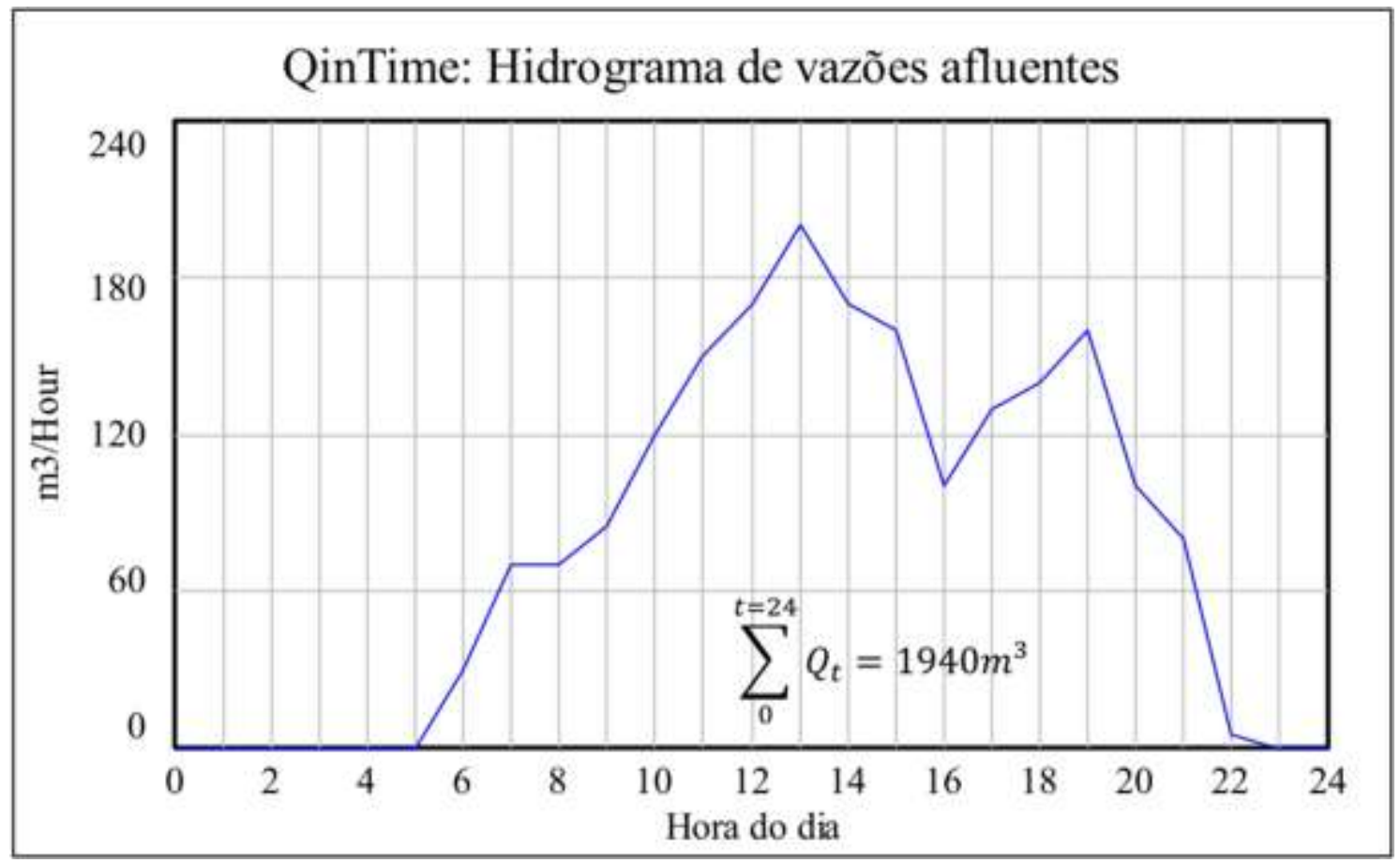

Figura 4 - Hidrograma de vazões afluentes para o presente trabalho

5. O enchimento correspondente à segunda batelada no reator 2 só poderá iniciar até que se tenha completado o enchimento para a segunda batelada no reator 1 e, além disso, se tenha finalizado o esvaziamento do efluente tratado na primeira batelada no reator 2. Se o esvaziamento ainda não tiver acabado, se segue um procedimento similar ao descrito no passo 4 , com relação à bacia de amortecimento e ao by-pass.

6. O volume armazenado na bacia de amortecimento é transferido para o reator 2, parcial ou totalmente (em dependência da vazão afluente simultânea), durante o enchimento correspondente à segunda batelada deste tanque. Para o presente trabalho se assumiu que este deslocamento é realizado a uma razão de $100 \mathrm{~m}^{3} / \mathrm{h}$ (definido no AuxValve). A transferência finaliza quando o volume máximo do reator (controlado por FillVol2) for atingido.

7. Depois de finalizado o enchimento do reator 2 , correspondente à segunda batelada, a vazão afluente ao sistema será armazenada na bacia de amortecimento; mas se esta não existir ou uma vez que atinge seu volume máximo, o esgoto não tratado será despejado através do by-pass. O volume armazenado na bacia de amortecimento no final do dia será transferido para o reator 1 , sendo o volume com que iniciará a primeira batelada no dia seguinte, segundo o já mencionado no passo 1 . No caso de existir um único tanque, o volume armazenado na bacia de amortecimento será o volume inicial para o dia seguinte no reator 2.

Uma vez descrita a lógica por trás do modelo, descrevem-se os elementos mostrados na Figura 3, utilizados para simular as bateladas: 
- Volume1a [m³]: Variável de acumulação (level variable) que representa o enchimento do reator 1 durante a primeira batelada. O volume com que começa o dia vai ser o volume armazenado na bacia de amortecimento no final do dia anterior (PrevDay). Seu valor máximo é definido através de FillVol1. No Vensim:

"Volume1a" = INTEG(IF THEN ELSE("Fill.Vol1">0.5 :AND: "Volume1a" <= "Fill.Vol1", "QinTime"+PrevDay, 0.001 ))

- Volume $2 a\left[\mathrm{~m}^{3}\right]$ : Variável de acumulação que representa o enchimento do reator 2 durante sua primeira batelada. No caso de existir um único tanque (representado por Fill.Voll < 0,5), o volume com que começa o dia vai ser o volume armazenado na bacia de amortecimento no final do dia anterior (PrevDay). Seu valor máximo é definido através de FillVol2.

"Volume2a" = INTEG (IF THEN ELSE( "Fill.Vol1" < 0.5 :AND: "Volume2a" <= "Fill.Vol2" :AND: "Fill.Vol2" > 0.5,

"QinTime"+PrevDay,

IF THEN ELSE("Volume1a" >= "Fill.Vol1" :AND: "Volume2a" <= "Fill.Vol2" :AND: "Fill. Vol2" > 0.5, "QinTime", 0)),0)

- Volume $1 b\left[\mathrm{~m}^{3}\right]$ : Variável de acumulação que representa o enchimento do reator 1 durante a segunda batelada, segundo o descrito no passo 4 da sequência lógica para modelagem das bateladas.

"Volume1b" = INTEG (IF THEN ELSE("Volume1a" >= "Fill.Vol1" :AND: "DecantVol1a" $>=$ "Fill.Vol1" :AND: "Volume2a" >= "Fill.Vol2" :AND: "Volume1b" <= "Fill.Vol1" :AND: "Fill.Vol1" > 0.5, "QinTime", 0.001 ), 0)

- Volume $2 b\left[\mathrm{~m}^{3}\right]$ : Variável de acumulação que representa o enchimento do reator 2 durante a segunda batelada, segundo o descrito nos passos 5 e 6 da sequência lógica para modelagem das bateladas.

"Volume2b" = INTEG (IF THEN ELSE("DecantVol2a" >= "Fill.Vol2" :AND: "Volume1b" $>=$ "Fill.Vol1" :AND: "Volume2b" < "Fill.Vol2" :AND: "Fill.Vol2" > 0.5, "QinTime" + "AuxValve",0 ), 0)

- DecantVol1a, DecantVol2a, DecantVol1b, DecantVol2b [m³]: Variáveis de acumulação para controlar o esvaziamento dos tanques, uma vez finalizado o processo de aeração e sedimentação do esgoto tratado. A razão de esvaziamento está controlada pelo elemento DelayVol correspondente. Usando como exemplo o DecantVol2a:

"DecantVol2a"= INTEG (IF THEN ELSE ("DecantVol2a"<= "Fill.Vol2", "DelayVol2a", 0), 0)

- DelayVol1a, DelayVol2a, DelayVol1b, DelayVol2b $\left[\mathrm{m}^{3} / \mathrm{h}\right]$ : Elementos do tipo retardo fixo (Delay Fixed) foram utilizados para controlar o retardo decorrente do período de aeração e sedimentação $(t A S)$ nos reatores para cada uma das bateladas. A lógica no Vensim é a seguinte: SE o volume acumulado for maior que a capacidade do tanque, $\mathrm{E}$ o volume liberado for menor que a capacidade do tanque, ENTÃO esvaziar o reator a uma razão definida por $D P R\left[\mathrm{~m}^{3} / \mathrm{h}\right]$ até atingir o valor da capacidade do tanque, SENÃO não esvaziar; o processo deverá ser retardado um valor de $t A S[h]$, com valor inicial de 0 . Usando como exemplo o DelayVol1b:

"DelayVol1b" = DELAY FIXED (IF THEN ELSE ("Volume1b" >= "Fill.Vol1" :AND: "DecantVol1b" <= "Fill.Vol1", DPR, 0), tAS,

- Retention $\left[\mathrm{m}^{3}\right]$ : Variável de acumulação que representa a bacia de amortecimento, segundo o explicado nos passos 4 ao 7 da sequência lógica para modelagem das bateladas. 
"Retention" = INTEG (

IF THEN ELSE("Fill.Vol1" > 0.5 :AND: "Retention" <= CapBasin

:AND:

(((“"DecantVol1a" <= "Fill.Vol1" :AND: "Volume1a" >= "Fill.Vol1") :AND: ("DecantVol2a"

$<=$ "Fill.Vol2" :AND: "Volume2a" >= "Fill.Vol2"))

:OR:

("DecantVol1b" <= "Fill.Vol1" :AND: "Volume1b" >= "Fill.Vol1") :AND: ("DecantVol2b" $<=$ "Fill.Vol2" :AND: "Volume2b" >= "Fill.Vol2")

:OR:

("DecantVol1b" <= "Fill.Vol1":AND: "Volume1b" >= "Fill.Vol1") :AND: ("DecantVol2a" $<=$ "Fill.Vol2" :AND: "Volume2a" >= "Fill.Vol2"))

:OR:

(("Volume1a":AND:"Volume1b">="Fill.Vol1") :AND: ("Volume2a":AND:"Volume2b">= "Fill.Vol2")))

, "QinTime"

, IF THEN ELSE ("Fill.Vol1" <= 0.5 :AND: "Retention" <= CapBasin :AND: (("DecantVol2a" $<=$ "Fill.Vol2" :AND: "Volume2a" >= "Fill.Vol2")

:OR:

("DecantVol2b" <= "Fill.Vol2" :AND: "Volume2b" >= "Fill.Vol2")

:OR:

("Volume2a" :AND: "Volume2b">"Fill.Vol2"))

, "QinTime",-"AuxValve")), 0)

- AuxValve $\left[\mathrm{m}^{3} / \mathrm{h}\right]$ : Elemento do tipo fluxo (rate) que permite realizar a transferência do volume armazenado na bacia de amortecimento para o reator 2, conforme o descrito no passo 6 da sequência lógica para modelagem das bateladas.

"AuxValve" = IF THEN ELSE ("Volume2b" > 0 :AND: ("Fill.Vol2" - "Volume2b" >=0) :AND: "Retention" > 0, 100,0 )

- Bypass [m3]: Variável de acumulação utilizada para controlar o volume diário que seria despejado diretamente pelo by-pass, sem passar pelo tratamento, segundo o descrito nos passos 4,5 e 7 da sequência lógica para modelagem das bateladas. Minimizar o valor desta variável deve ser um dos objetivos do dimensionamento e regime de operação do sistema.

"Bypass" = INTEG (

IF THEN ELSE("Fill.Vol1" > 0.5 :AND: "Retention" > CapBasin :AND: (((“"DecantVolla" $<=$ "Fill.Vol1" :AND: "Volume1a" >= "Fill.Vol1") :AND: ("DecantVol2a" <= "Fill.Vol2" :AND: "Volume2a" >= "Fill.Vol2"))

:OR:

("DecantVol1b" <= "Fill.Vol1":AND: "Volume1b" >= "Fill.Vol1") :AND: ("DecantVol2b" $<=$ "Fill.Vol2" :AND: "Volume2b" >= "Fill.Vol2")

:OR:

("DecantVol1b" <= "Fill.Vol1" :AND: "Volume1b" >= "Fill.Vol1") :AND: ("DecantVol2a" $<=$ "Fill.Vol2" :AND: "Volume2a">="Fill.Vol2"))

:OR:

("“Volume1a":AND:"Volume1b">="Fill.Vol1") :AND: ("Volume2a":AND:"Volume2b">= "Fill.Vol2")))

, "QinTime"

,IF THEN ELSE ("Fill.Vol1" <=0.5 :AND: "Retention" > CapBasin :AND: (("DecantVol2a" $<=$ "Fill.Vol2" :AND: "Volume2a" >= "Fill.Vol2")

:OR:

("DecantVol2b" <= "Fill.Vol2" :AND: "Volume2b">="Fill.Vol2")

:OR: 
("Volume2a" :AND: "Volume2b">"Fill.Vol2"))

, "QinTime-3", 0 )), 0)

- TotalVolDay $\left[\mathrm{m}^{3}\right]$ : Variável auxiliar utilizada para acompanhar a contagem do volume total tratado por dia, isto é, aquele que passa pelos reatores.

"TotalVolDay" = "Volume1a"+"Volume1b"+"Volume2a"+"Volume2b"

- Tank1, Tank2 [m³]: Variáveis auxiliares para dar seguimento ao comportamento dos volumes que passam pelo reator correspondente. Usando como exemplo o Tank1:

Tank1 = Max("Volume1a" - "DecantVol1a",0) + Max("Volume1b" - "DecantVol1b",0)

- Qsbr [m3]: Somatória de Tank1 e Tank2, para representar o comportamento dos volumes de esgoto tratados no sistema.

- PrevDay: Variável auxiliar que serve para trasladar, desde a bacia de amortecimento, o volume armazenado ao final do dia anterior para incluí-lo na primeira batelada do dia. Por exemplo, para o terceiro dia corresponde o PrevDay2:

PrevDay2 = IF THEN ELSE (Time >= 48 :AND: Time<= 49 :AND: "Retention-2" >0, "Retention-2", 0 )

Foi adicionada, também, uma vista para permitir ao usuário realizar análises de sensibilidade, a qual se apresenta na Figura 5. Nela se podem controlar as variáveis que definem o comportamento do sistema e verificar como mudanças nestes valores afetam a fração de esgoto tratado e a fração que se despejaria diretamente, sem tratar, através do by-pass.

Uma vez finalizada a descrição do modelo feito no Vensim, deve-se recordar que existem outros programas de software (e.g.: Powersim, Stella), nos quais, por meio da utilização de dinâmica de sistemas e considerações similares às do presente trabalho, seria possível representar sistemas similares.

Igualmente, e como já foi mencionado na seção introdutória, o objetivo deste trabalho é servir como base no desenvolvimento de modelos que considerem os efeitos que os padrões de enchimento e esvaziamento nos reatores em bateladas sequenciais têm na eficiência do tratamento e operação do sistema, a través da adição de outros elementos ao modelo (transferência de oxigênio, concentrações de carga, parâmetros cinéticos), em função das particularidades do sistema que representam.

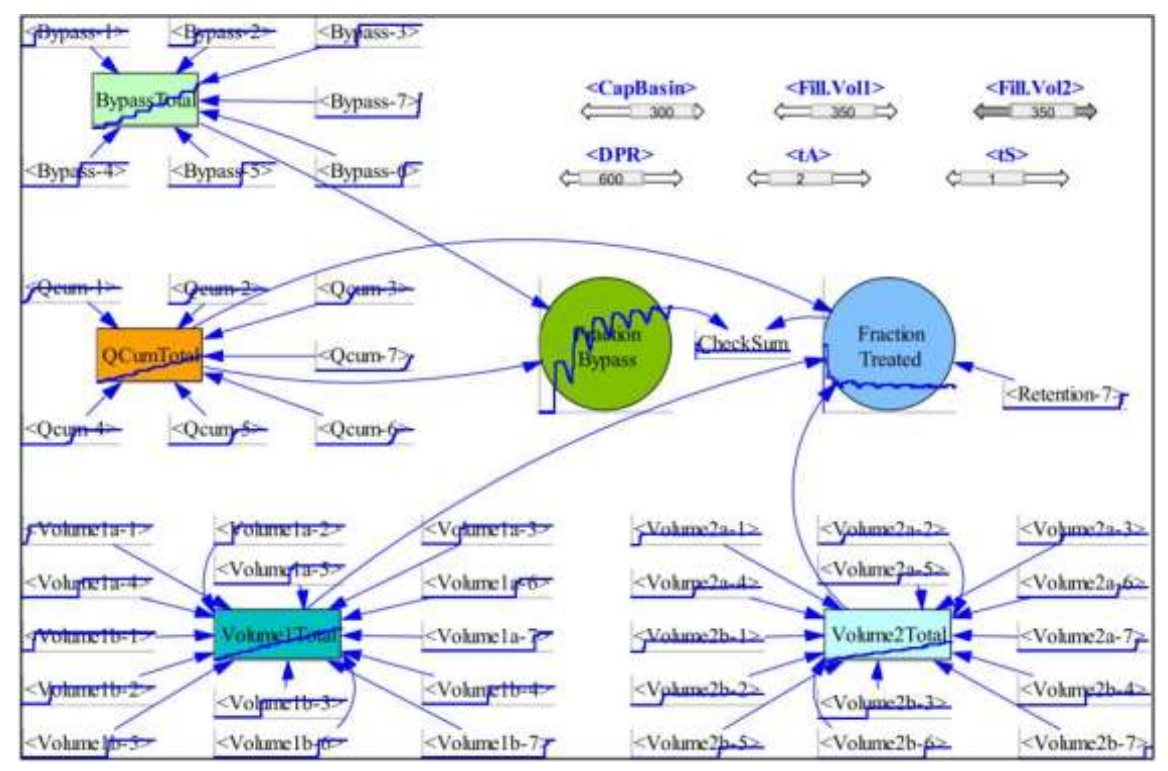

Figura 5 - Vista no Vensim para realizar análises de sensibilidade no modelo 


\section{EXEMPLOS: RESULTADOS E DISCUSSÃO}

O presente documento tem como propósito descrever um modelo de dinâmica de sistemas, feito no Vensim, representando o enchimento e o esvaziamento dos tanques, em uma estação de tratamento de esgoto, com reatores em bateladas sequenciais. Alguns exemplos são incluídos para ilustrar a capacidade do modelo de simular diferentes condições de configuração do sistema.

Uma aplicação do modelo se relaciona com o momento de projetar o sistema de aeração e seu regime de operação, no qual as variações diurnas das cargas a remover são um fator importante a considerar. Um exemplo disto é citado por Flanagan e Bracken (1977). Nesse texto se menciona o caso de uma estação de tratamento na Carolina do Norte, em que a vazão de esgoto afluente apresentava concentrações de Nitrogênio total que variavam entre $26 \%$ e $216 \%$ com relação à média ao longo de um período de $24 \mathrm{~h}$, e as de DBO entre $13 \%$ e $190 \%$. A consideração de uma vazão constante subestimaria ou superestimaria a massa total das diferentes cargas a remover. Nesse contexto, a dinâmica de sistemas e um modelo similar ao apresentado neste trabalho poderiam resultar úteis na avaliação de diferentes cenários de tratamento. Para isto, bastariam algumas modificações específicas ao sistema representado, em função dos tipos de cargas a remover, parâmetros cinéticos, químicos e físicos da estação de tratamento.

Para verificar que o modelo é capaz de representar as características e comportamentos explicados na sua descrição, nesta seção são apresentados três exemplos, considerando configurações diferentes:

1. Dois tanques sem bacia de amortecimento

2. Dois tanques com bacia de amortecimento

3. Um único tanque

Nestes exemplos, os reatores 1 e 2 têm o mesmo volume de zona de carga quando o sistema contém dois tanques. Duas opções de volume foram consideradas $\left(450 \mathrm{~m}^{3}\right.$ e $\left.500 \mathrm{~m}^{3}\right)$ para avaliar seu efeito na capacidade de tratamento para 2 ou 4 bateladas por dia. O volume utilizado para a bacia de amortecimento, quando incluída, é de $200 \mathrm{~m}^{3}$.

Os valores de outros parâmetros importantes utilizados no presente trabalho são:

- Tempo de aeração/sedimentação $(t A S)$ :

$3 \mathrm{~h}$

o Tempo de aeração $(t A)$ :

$2 \mathrm{~h}$

- Tempo de sedimentação $(t S)$ :

- Capacidade das bombas (DPR):

$1 \mathrm{~h}$

$600 \mathrm{~m}^{3} / \mathrm{h}$

\section{I EXEMPLO I: DOIS TANQUES SEM BACIA DE AMORTECIMENTO}

Com dois tanques é possível realizar até quatro bateladas por dia. O anterior significa que dois reatores de $450 \mathrm{~m}^{3}$ teriam a capacidade de tratar até $1800 \mathrm{~m}^{3}\left(92,8 \%\right.$ do volume diário de $\left.1940 \mathrm{~m}^{3}\right)$ e dois reatores de $500 \mathrm{~m}^{3}$ poderiam atender a totalidade da vazão diária afluente ao sistema. No entanto, as vazões afluentes não seguem um comportamento uniforme, razão pela qual o enchimento de cada tanque, em cada batelada, depende do regime de lançamento do esgoto.

Na Figura 6 se apresentam os resultados de um dia para o caso em que se analisam dois tanques com volume de zona de carga de $450 \mathrm{~m}^{3}$, sem bacia de amortecimento, para a vazão afluente da Figura 4. A partir das quatro linhas tracejadas na Figura 6, é possível observar o seguinte: 1) O enchimento para a primeira batelada no tanque 2 só começa quando o tanque 1 estiver cheio; 2) O esgoto é despejado diretamente através do by-pass se os tanques não estiverem disponíveis para armazenar (durante o tempo de aeração/sedimentação ou esvaziando volume tratado); 3) O despejo através do by-pass é interrompido uma vez que algum dos tanques tiver a capacidade de armazenar de novo o esgoto afluente; 4) $\mathrm{O}$ início da segunda batelada no tanque 2 segue a mesma lógica que em 1).

Cabe lembrar que, segundo o mencionado anteriormente nas considerações do modelo, se o tanque 2 não atinge seu volume máximo ao final do dia, considera-se que o volume com que finaliza o dia é tratado e liberado antes do início da primeira batelada nesse reator no dia seguinte.

Para a condições avaliadas neste cenário, o volume diário tratado seria de $1736 \mathrm{~m}^{3}(89,5 \%$ do volume diário de $1940 \mathrm{~m}^{3}$ ) e, consequentemente, aquele que seria despejado através do by-pass de $204 \mathrm{~m}^{3}$, 
significando um tratamento menor ao $92,8 \%$ do esperado, em função do volume dos tanques.

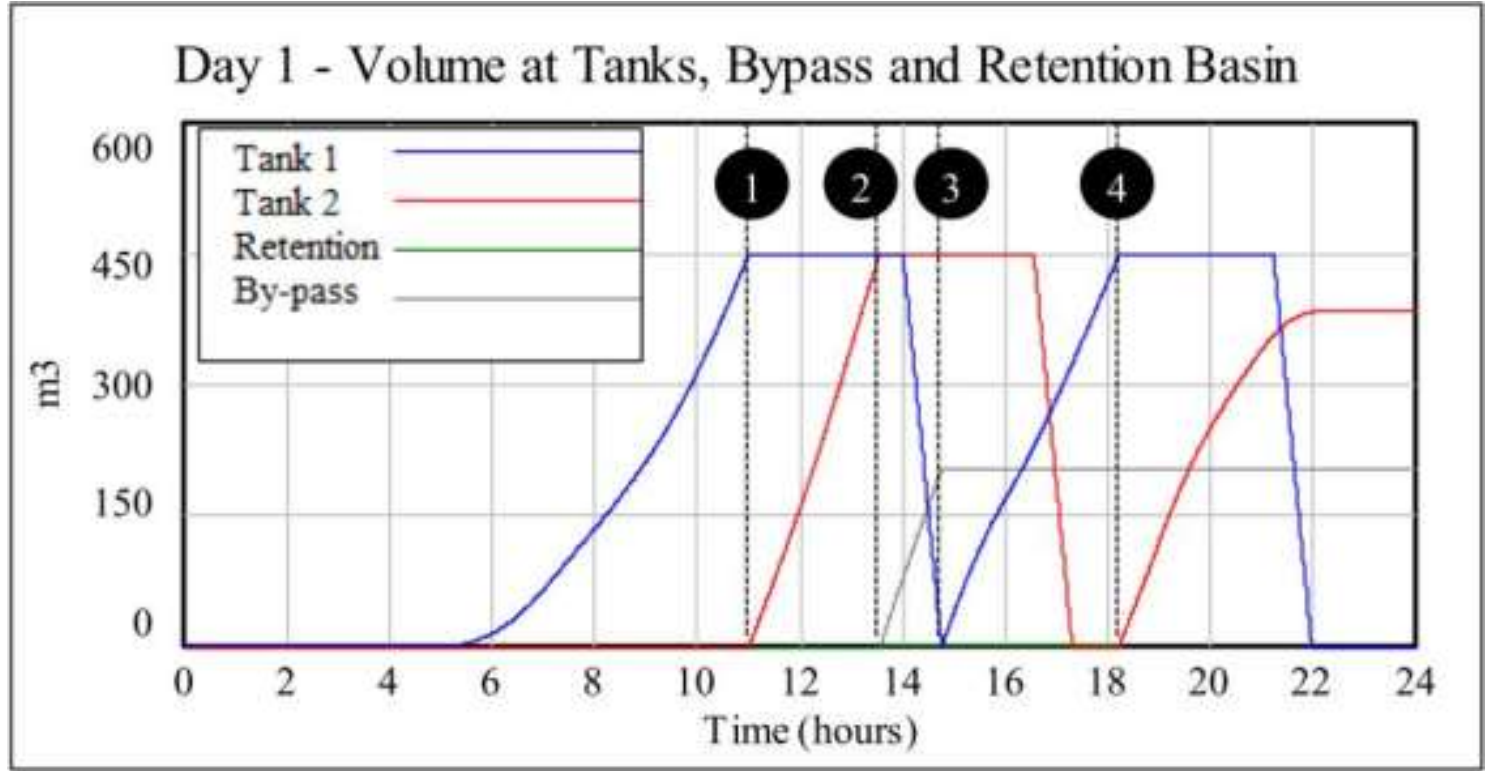

Figura 6 - Resultados durante 1 dia para 2 tanques de $450 \mathrm{~m}^{3}$, sem bacia de amortecimento

Na Figura 7 se apresentam os resultados de um dia para o caso em que se analisam dois tanques com volume de zona de carga de $500 \mathrm{~m}^{3}$ sem bacia de amortecimento. Por causa do volume dos reatores, eles seriam capazes de tratar até $2000 \mathrm{~m}^{3}$ em função das 4 bateladas; porém, conforme se observa na Figura 7, o regime de vazão afluente e os tempos de aeração e sedimentação causariam que parte da vazão fosse despejada diretamente, sem tratar, através do by-pass. Segundo os resultados mostrados na figura para as condições avaliadas neste caso, dos $1940 \mathrm{~m}^{3}$ do volume de esgoto diário, até $1772 \mathrm{~m}^{3}$ $(91,4 \%)$ poderiam ser tratados e $168 \mathrm{~m}^{3}(8,6 \%)$ seriam despejados diretamente através do by-pass. Estes resultados evidenciam a importância de considerar uma bacia de amortecimento.

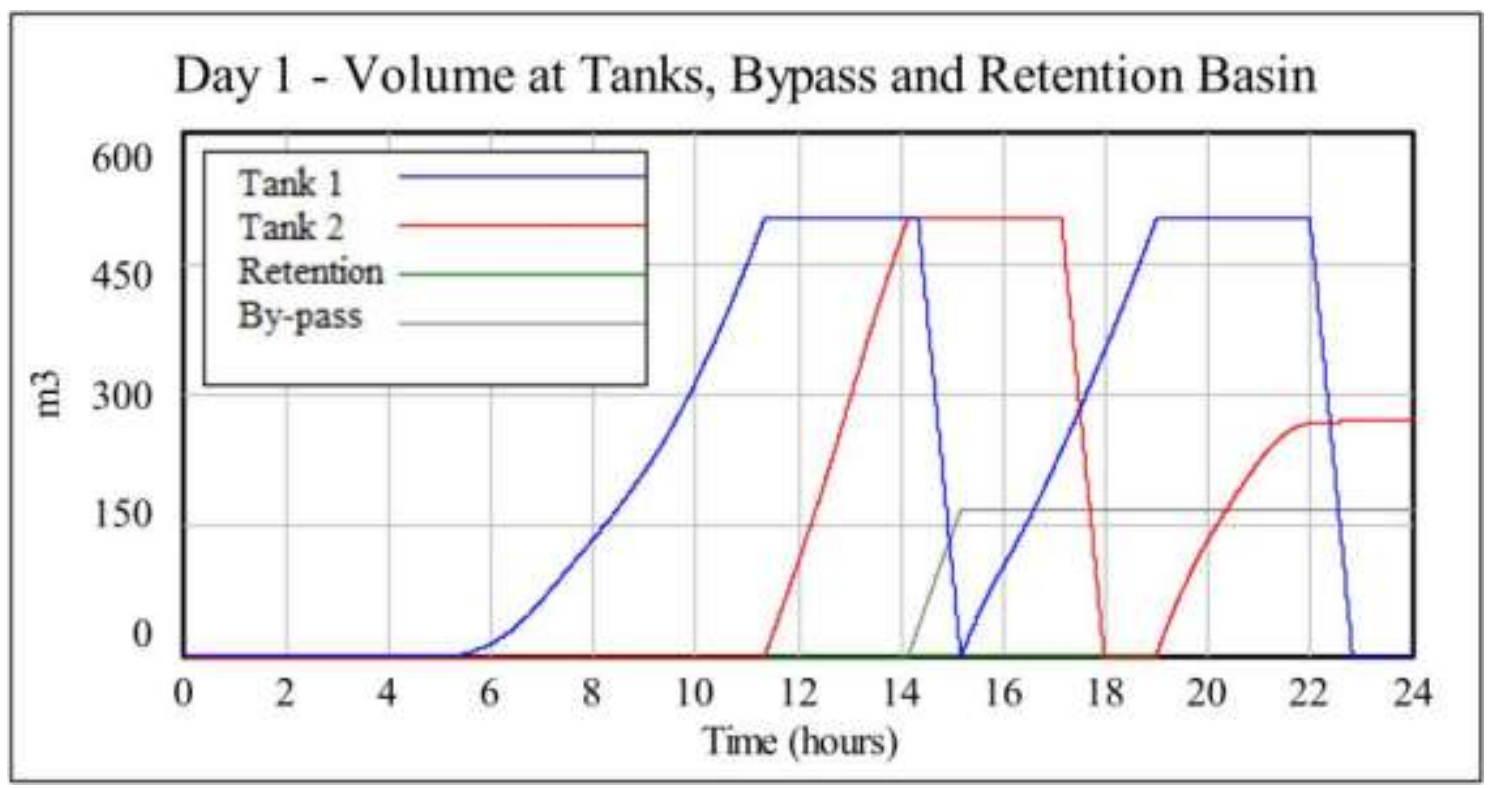

Figura 7 - Resultados durante 1 dia para 2 tanques de $500 \mathrm{~m}^{3}$, sem bacia de amortecimento 


\subsection{EXEMPLO 2: DOIS TANQUES COM BACIA DE AMORTECIMENTO}

Neste exemplo, considera-se uma configuração do sistema na qual se inclui uma bacia de amortecimento de $200 \mathrm{~m}^{3}$, para avaliar o efeito que esta poderia ter no volume de esgoto tratado, quando trabalha em conjunto com os tanques do exemplo 1.

Para um sistema formado por dois tanques de $450 \mathrm{~m}^{3}$, bacia de amortecimento de $200 \mathrm{~m}^{3}$ e vazão afluente diária de esgoto mostrada na Figura 4, apresentam-se duas figuras que permitem explicar o comportamento do sistema para estas condições, obtidas a partir dos resultados do modelo. Na Figura 8 se mostra o comportamento esperado dos volumes acumulados tanto do tratamento nos tanques quanto de volume despejado através do by-pass. Nesta figura é possível observar que, para estas condições e durante as primeiras $45 \mathrm{~h}$, o conjunto dos reatores com a bacia de amortecimento seria capaz de tratar a totalidade do esgoto, sem ser necessário despejar através do by-pass. Ao final do período de $168 \mathrm{~h}$ da simulação, dos $13580 \mathrm{~m}^{3}$ afluentes ao sistema, $780 \mathrm{~m}^{3}$ (5,7\%) seriam despejados sem tratar. Portanto, a inclusão da bacia de amortecimento permitiria tratar $94,3 \%$ do esgoto afluente, em comparação dos $89,5 \%$ sem esta estrutura.

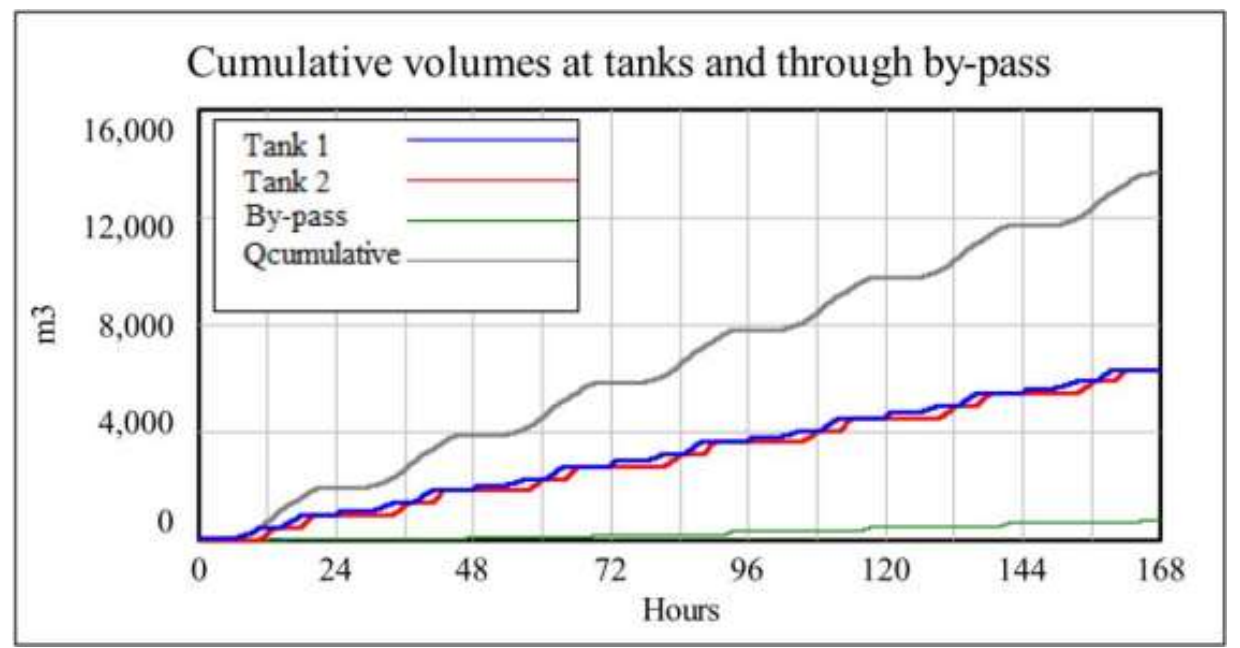

Figura 8 - Dois tanques de $450 \mathrm{~m}^{3}$ com bacia de amortecimento: Volumes acumulados

A segunda imagem utilizada para explicar o comportamento do sistema descrito é a Figura 9 , na qual se apresenta o comportamento do sistema durante o quarto dia da simulação (entre as $72 \mathrm{~h}$ e às 96h). Por meio das linhas tracejadas nessa figura, é possível observar os seguintes aspectos relacionados com a bacia de amortecimento: 1) Durante o início do dia, o volume armazenado na bacia

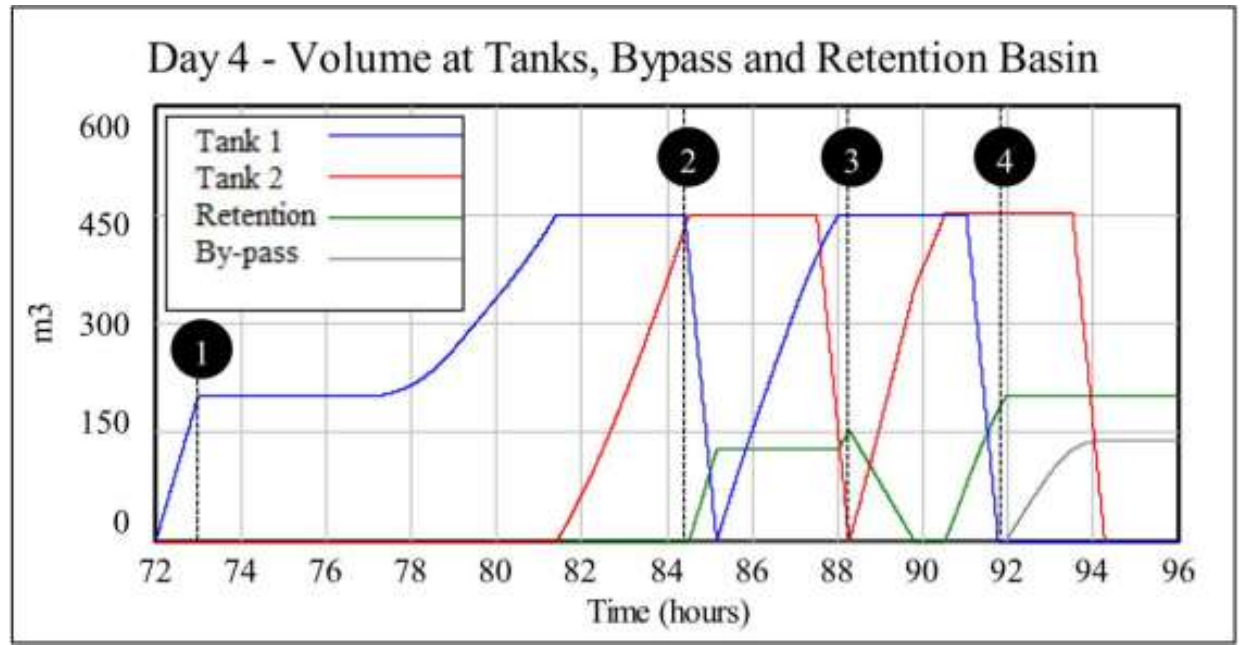

Figura 9 - Resultados durante 1 dia para 2 tanques de $450 \mathrm{~m}^{3}$, com bacia de amortecimento 
ao final do dia anterior é transferido para o tanque 1, como volume inicial da primeira batelada; 2) A bacia de amortecimento começa a armazenar se os tanques não estiverem disponíveis para armazenar (durante o tempo de aeração/sedimentação ou esvaziando volume tratado); 3) O volume armazenado na bacia de amortecimento é incluído durante o enchimento da segunda batelada do reator 2; 4) O esgoto só será despejado através do by-pass quando a bacia de amortecimento atingir seu volume máximo. Pela forma em que foi concebida e modelada a operação do sistema, só foram projetadas, como máximo, duas bateladas por tanque por dia, mas é fácil notar que, em lugar de utilizar o by-pass, as vazões excedentes poderiam ter sido recebidas pelo reator 1. Aplicando os princípios descritos no presente documento, pode-se modificar o modelo para incluir uma ou mais bateladas adicionais, o que incrementaria a capacidade de tratamento do sistema.

Quando se considera um sistema formado por dois tanques de $500 \mathrm{~m}^{3}$, com bacia de amortecimento de $200 \mathrm{~m}^{3}$ e vazão afluente diária mostrada na Figura 4, os resultados obtidos a partir do modelo sugerem que, seguindo as condições de operação descritas no presente trabalho, seria possível tratar $100 \%$ do esgoto afluente. Estes resultados se exibem na Figura 10, demonstrando, novamente, a vantagem de incluir uma bacia de amortecimento no leiaute do sistema.

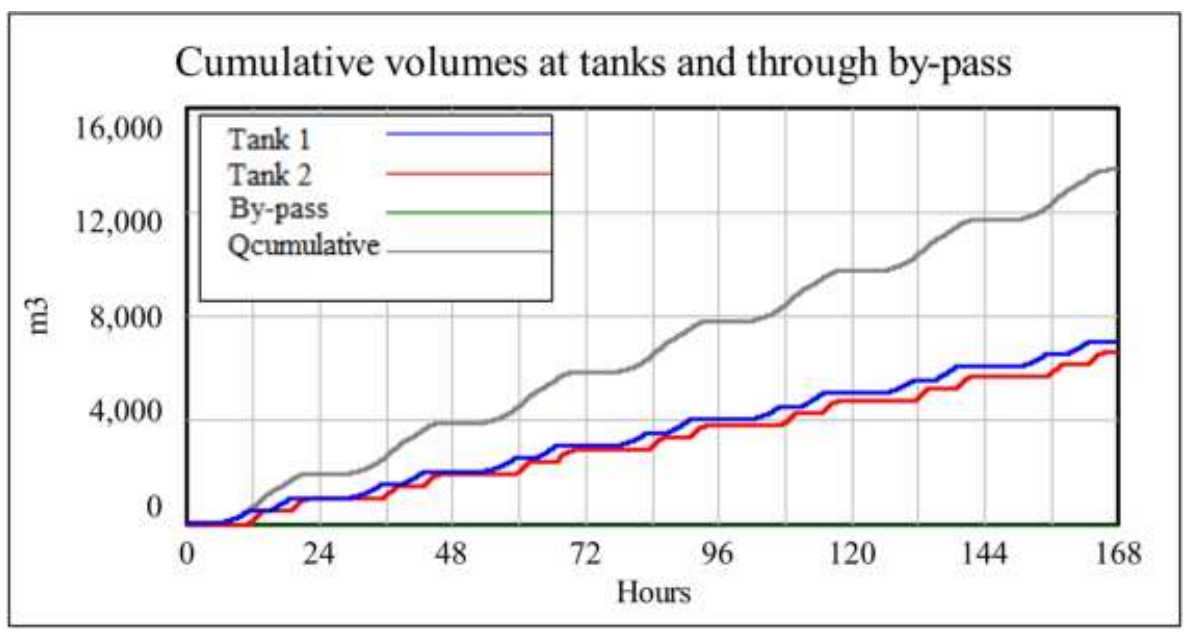

Figura 10 - Dois tanques de $500 \mathrm{~m}^{3}$ com bacia de amortecimento: Volumes acumulados

\subsection{EXEMPLO 3: UM ÚNICO TANQUE}

Em função de variações sazonais na vazão afluente, às vezes é útil retirar da operação, temporalmente, um dos reatores, com o propósito de minimizar os custos associados e realizar atividades de manutenção (NEIWPCC, 2005). Neste terceiro exemplo, avalia-se o comportamento do sistema modelado com um único tanque com bacia de amortecimento, para uma vazão reduzida em $50 \% \mathrm{com}$ relação à mostrada na Figura 4 e considerando dois volumes de zona de carga diferentes para o reator $\left(450 \mathrm{~m}^{3}\right.$ e $\left.500 \mathrm{~m}^{3}\right)$. Na Figura 11 se apresentam, de forma conjunta, os resultados para este sistema que considera essas duas condições de volume na zona de carga. Igualmente, na mesma figura, inclui-se o volume acumulado, que seria despejado através do by-pass, caso não fosse utilizada uma bacia de amortecimento para o caso de um tanque com volume de carga de $500 \mathrm{~m}^{3}$.

Quando se considera o uso de uma bacia de amortecimento de $200 \mathrm{~m}^{3}$, e a partir do mostrado na Figura 11, observa-se que os $50 \mathrm{~m}^{3}$ de diferença no volume de carga (incremento de $11 \%$ com relação ao volume de $450 \mathrm{~m}^{3}$ ) não implica um aumento significativo no volume que poderia ser tratado, já que, depois de um período de 168 horas, um tanque de $500 \mathrm{~m}^{3}$ só conseguiria tratar $0,88 \%$ a mais do que o tanque com volume menor. Portanto, para ambos cenários, o volume despejado através do by-pass seria de menos de $500 \mathrm{~m}^{3}$, o que poderia ser facilmente tratado realizando uma ou duas bateladas adicionais na semana, seja no mesmo reator, seja no tanque que está temporalmente fora da operação.

Se a bacia de amortecimento não estivesse incluída na configuração do sistema, mesmo para o caso de um tanque com volume de carga de $500 \mathrm{~m}^{3}$ o volume despejado diretamente, sem tratar, seria de $1810 \mathrm{~m}^{3}, 27 \%$ do volume total para o período analisado de 168 horas. Portanto, mais uma vez, fica evidente a utilidade desta estrutura. 
Para finalizar esta seção, é conveniente mencionar que a versão profissional do Vensim permite otimizar valores, tais como a capacidade das bombas, os volumes dos reatores ou da bacia de amortecimento, a fim de minimizar a fração do volume sem tratar. Porém, esta característica não foi utilizada no presente trabalho.

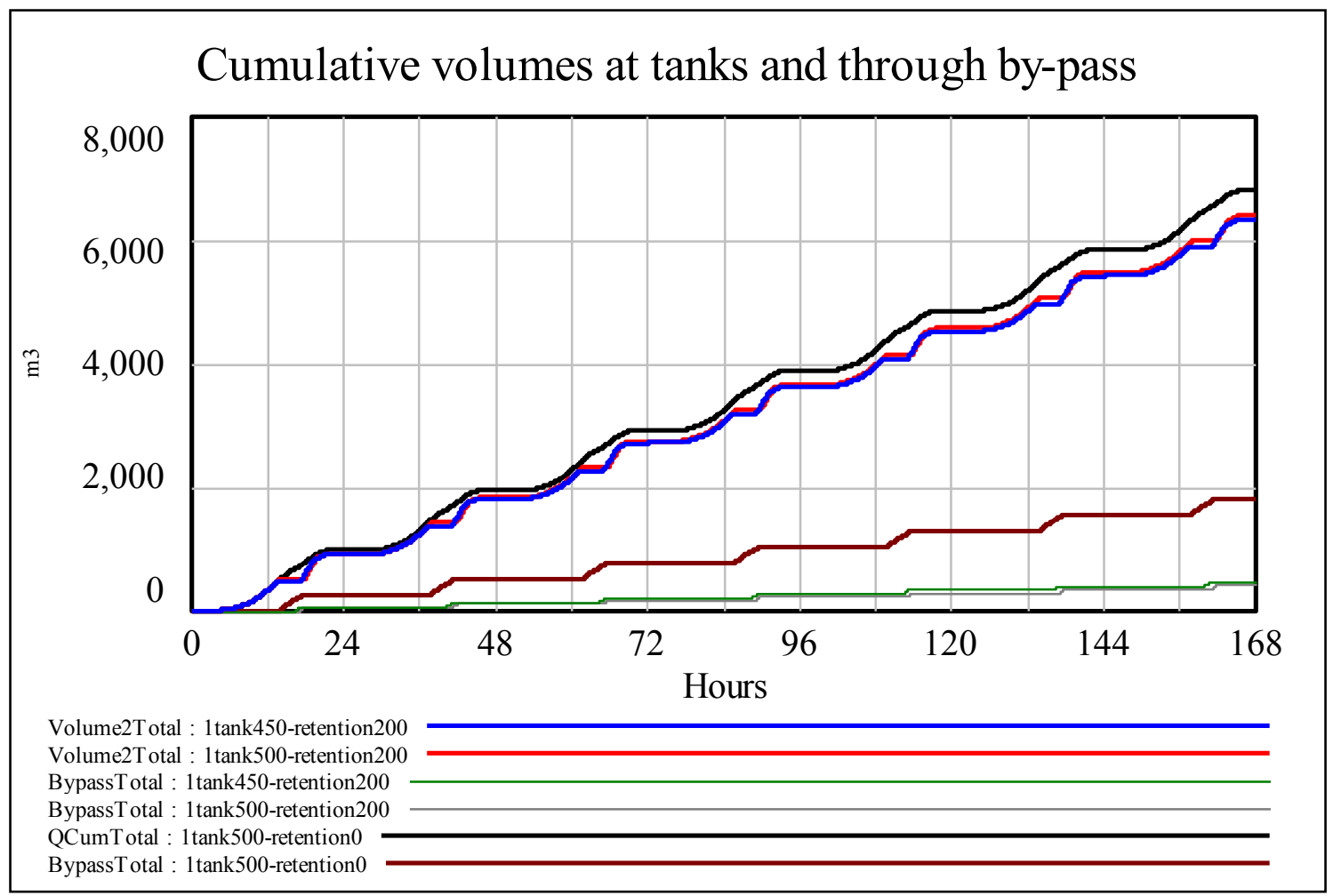

Figura 11 - Um único tanque de $450 \mathrm{~m}^{3}$ ou $500 \mathrm{~m}^{3}$ : Volumes acumulados

\section{CONCLUSÕES}

No presente documento foi apresentado um modelo, baseado em dinâmica de sistemas, que permite representar o enchimento e o esvaziamento de reatores, em bateladas sequenciais, para tratamento de esgoto. O programa utilizado na criação do modelo foi o Vensim, mas outros programas poderiam ter sido utilizados para este propósito (e.g.: Stella, Powersim, etc.). Entre os benefícios de usar a dinâmica de sistemas, podem ser mencionados: 1) Permite realizar de múltiplos cálculos de forma simultânea em modelos que podem ser muito complexos; 2) A interface gráfica dos principais programas disponíveis facilita sua aprendizagem, assim como a elaboração e interpretação das relações entre os elementos dos modelos.

Com base nos resultados dos exemplos mostrados, é possível observar que o modelo apresentado tem a capacidade de simular o funcionamento do sistema descrito para diferentes configurações. Igualmente, se ressalta que é possível modificar e adaptar o modelo, a fim de incluir características próprias de sistemas de tratamento similares, permitindo, assim, avaliar particularidades em função do tipo de esgoto a ser tratado e características físicas ou químicas da estação de tratamento.

Os resultados dos exemplos apresentados também permitem ilustrar que o comportamento não uniforme e os picos da vazão afluente afetam o tempo total de cada batelada, pelo qual se deveria ter cuidado quando se considera uma vazão de esgoto afluente constante, como geralmente se aplica nos métodos tradicionais. Outra conclusão importante que pode ser elaborada com base nos resultados obtidos é que, para maximizar o volume de esgoto tratado, é importante incluir uma bacia de amortecimento, caso contrário parte do esgoto poderia ser despejado, diretamente, ao corpo receptor, sem nenhum tipo de tratamento, ou bem seria necessário construir outro tanque ou aumentar a capacidade dos existentes. 
Finalmente, ainda que exista a necessidade de adaptar o presente modelo para que possa simular todas as características de uma estação de tratamento, julga-se que o modelo proposto, mesmo no atual estágio de desenvolvimento, constitui uma útil ferramenta para servir como base no desenvolvimento de representações de sistemas mais complexos ou com diferente configuração, apoiando, assim, no planejamento e na operação de reatores em bateladas sequenciais.

\section{AGRADECIMENTOS}

O primeiro autor gostaria de agradecer à CAPES pela bolsa de estudos que lhe permite realizar estudos de pós-graduação.

\section{REFERÊNCIAS}

AL-REKABI, W. S.; QIANG, H.; QIANG, W. W. Review on sequencing batch reactors. Pakistan Journal of nutrition, v. 6(1), p. 11-19, 2007.

FLANAGAN, M. J.; BRACKEN, B. D. Design procedures for dissolved oxygen control of activated sludge processes. Municipal Environmental Research Laboratory, Office of Research and Development, US Environmental Protection Agency. Cincinnaty, 1977.

FORD, F. A. Modeling the environment: an introduction to system dynamics models of environmental systems. 2. ed. Washington D.C.: Island Press, 2009. 400 p.

GONÇALVES, J. C. S. I. Desenvolvimento de modelo numérico para a simulação da qualidade da água em rios utilizando o software Vensim PLE. Dissertação (Mestrado em Engenharia Civil), Universidade de São Paulo, 2009.

IZQUIERDO, L. R.; GALÁN, J. M.; SANTOS, J. I.; OLMO, R. D. Modelado de sistemas complejos mediante simulación basada en agentes y mediante dinámica de sistemas. EMPIRIA. Revista de Metodología de Ciencias Sociales, n. 16, p. 85-112, 2008.

LIU, Y.; GUO, H.; YU, Y.; DAI, Y.; ZHOU, F. Ecological-economic modeling as a tool for watershed management: a case study of Lake Qionghai watershed, China. Limnologica-Ecology and Management of Inland Waters, 38(2), p. 89-104, 2008.

LOPES, F. A. (2011). Drenagem ácida de minas concentrada como fonte de ferro para a coagulação e para a Reação de Fenton: aplicações no tratamento de esgoto sanitário de uma instituição de ensino superior. Dissertação (Mestrado em Engenharia de Minas, Metalurgia e dos Materiais), Universidade Federal do Rio Grande do Sul, 2011.

METCALF \& EDDY, INC. Wastewater engineering: treatment and reuse. 4. ed. New York: McGraw-Hill, 2003. 1848 p.

NEW ENGLAND INTERSTATE WATER POLLUTION CONTROL COMMISSION. Sequencing Batch Reactor Design and Operational Considerations. Lowell, MA, 2005.

NOVOTNY, V.; IMHOFF, K. R.; OLTHOF, M.; KRENKEL, P. A. Handbook of urban drainage and wastewater. Hoboken, NJ : John Wiley \& Sons Inc., 1989.

SANTOS, R. C. O.; BARBOSA FILHO, O.; GIORDANO, G. Proposta de um método racional para o dimensionamento de reatores de tratamento de esgotos por lodos ativados em bateladas para remoção de carga orgânica. Eng. sanit. ambient, v. 11, n. 2, p. 153-161, abr/jun 2006. 
SOUZA, C. C. D. Estudo do desempenho de reatores de lodos ativados de fluxo contínuo e de batelada no tratamento de águas residuárias em regime não estacionário, Trabalho de Diplomação (Graduação em Engenharia Civil), Universidade Federal do Rio Grande do Sul, 2011.

STAVE, K. A. A system dynamics model to facilitate public understanding of water management options in Las Vegas, Nevada. Journal of Environmental Management, v. 67, n. 4, p. 303-313, 2003.

UNITED STATES ENVIRONMENTAL PROTECTION AGENCY. Wastewater Technology Fact Sheet Sequencing Batch Reactors. EPA 832-F-99-073. Washington D.C., 1999.

WEI, S.; YANG, H.; SONG, J.; ABBASPOUR, K. C.; XU, Z. System dynamics simulation model for assessing socio-economic impacts of different levels of environmental flow allocation in the Weihe River Basin, China. European Journal of Operational Research, v. 221, n. 1, p. 248-262, 2012. 